\title{
DEBRIS DISKS EXPLORED BY AKARI AND IRSF
}

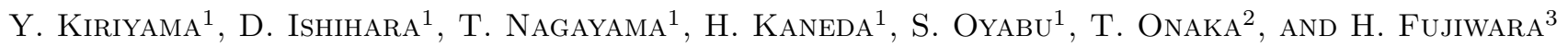 \\ ${ }^{1}$ Graduate School of Science, Nagoya University, Furo-cho, Chikusa-ku, Nagoya, Aichi, 464-8602, Japan \\ ${ }^{2}$ Graduate School of Science, University of Tokyo, 7-3-1, Hongo, Bunkyo-ku, Tokyo, 113-0033, Japan \\ ${ }^{3}$ Subaru Telescope, National Astronomical Observatory of Japan, 650 North A'ohoku Place, Hilo, HI96720, USA \\ E-mail: ishihara@u.phys.nagoya-u.ac.jp \\ (Received June 30, 2012; Accepted August 20, 2012)
}

\begin{abstract}
Using the AKARI mid-infrared all-sky survey catalogue, we are searching for debris disks which are important objects as an observational clue to on-going planetary system formation. Debris disk candidates are selected through a significant excess of the measured flux over the predicted flux for the stellar photospheric emission at $18 \mu \mathrm{m}$. The fluxes were originally estimated based on the near-infrared spectral energy distributions (SEDs) of central stars constructed from the 2MASS J-, H-, and Ks-band fluxes. However, we found that in many cases the 2MASS photometry has large errors due to saturation in the central part of a star image. Therefore we performed follow-up observations with the IRSF $1.4 \mathrm{~m}$ near-infrared telescope in South Africa to obtain accurate fluxes in the $\mathrm{J}-, \mathrm{H}-$, and Ks-bands. As a result, we have succeeded in improving the SEDs of the central stars. This improvement of the SEDs allows us to make more reliable selection of the candidates.
\end{abstract}

Key words: infrared; stars: infrared; planetary systems: surveys

\section{INTRODUCTION}

Debris disks are important objects as observational clues to planetary system formation. They were first detected by the IRAS survey. Since then, their number has increased by space-borne observations and their properties have been investigated by statistical studies (e.g. Wyatt et al., 2005). However, the critical evidence that links debris disks and planetary systems is still missing.

The AKARI mid-infrared (MIR) all-sky survey point source catalogue (PSC; Ishihara et al., 2010) potentially contains a large number of previously unrecognized debris disks. This unbiased survey can cover field stars in a larger survey volume, in contrast to the Spitzer Legacy programs, which concentrate on clusters or nearby stars. Furthermore, the AKARI/MIR survey has a potential to detect a new kind of debris disks that provide precious information on the history of colliding material (e.g. Fujiwara et al., 2012).
Debris disks are detected with a significant excess of the measured flux over the predicted flux for the stellar photospheric emission at $18 \mu \mathrm{m}$. The MIR fluxes of the central stars have been originally estimated based on the near-infrared (NIR) spectral energy distributions (SEDs) of central stars constructed from the 2MASS J, H-, and Ks-band fluxes. However, in many cases, the 2MASS photometry has large errors due to saturation in the central part of a star image. Accurate estimate of the fluxes of central stars would enable us to make reliable detections and accurate estimates of the MIR excess components. Therefore, we perform follow-up observations with the Infrared Survey Facility (IRSF) $1.4 \mathrm{~m}$ NIR telescope in South Africa to obtain accurate fluxes in the $\mathrm{J}-, \mathrm{H}-$, and Ks-bands, adopting neutral density (ND) filters to attenuate high fluxes. 


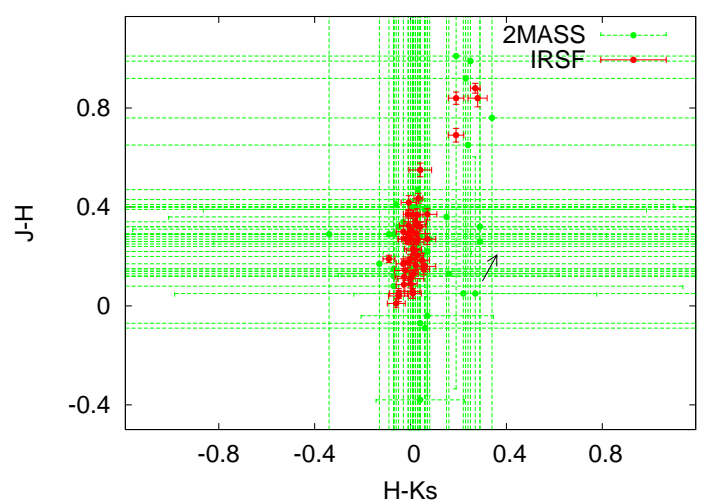

Fig. 1. $[\mathrm{J}]-[\mathrm{H}]$ vs. $[\mathrm{H}]-[\mathrm{Ks}]$ color-color diagram for our sample. The red points indicate IRSF observations while the green points indicate 2MASS observations. The reddening vector of $A_{v}=1$ is indicated by the arrow.

\section{OBSERVATION AND DATA ANALYSIS}

\subsection{Sample Selection}

First, we made a list of dwarfs to be investigated. We cross-matched the AKARI $18 \mu \mathrm{m}$ sources with dwarfs in the Tycho-2 spectral catalogue (Wright et al., 2003) simply by position. The search radius was set to 3 arc-seconds, and 1,735 sources were matched. Then, we checked the reliability of the cross-IDs by images. We also confirmed the spectral type in the SIMBAD database. We removed suspicious multiple stars, YSOs, mass-losing stars, and variable stars. As a result, we selected 734 secure dwarfs, which have AKARI $18 \mu \mathrm{m}$ fluxes.

\subsection{IRSF Observation}

Among our sample, we made J-, H-, and Ksphotometry of 165 stars on 2011 August 6 and 2011 August 9 using the IRSF telescope with the ND filters.

Figure 1 shows the $[\mathrm{J}]-[\mathrm{H}]$ versus $[\mathrm{H}]-[\mathrm{Ks}]$ color-color plot of our sample together with the intrinsic colors of dwarfs. Compared with the 2MASS photometry, the IRSF photometric results greatly improve the accuracy and the reliability of the fluxes of the central stars.

\section{RESULTS}

For the already observed 165 stars, we compared the AKARI $18 \mu \mathrm{m}$ fluxes with the fluxes of the central stars predicted from our IRSF NIR photometry and the model photosphere (Kurucz, 2003). Figure 2 demonstrates how the accuracy is improved in the detection

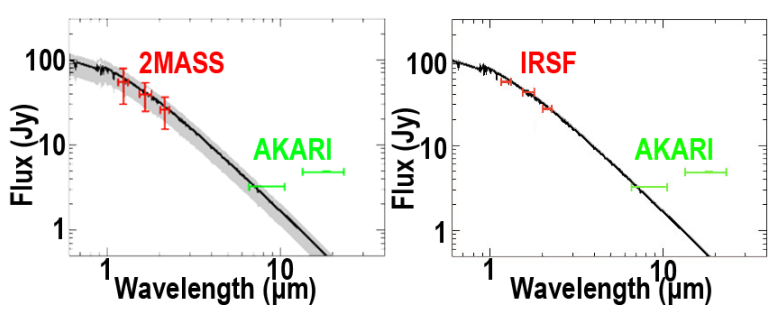

Fig. 2. Examples of SED fitting of a central star where photospheric emission is derived by 2MASS (left) and IRSF observations (right).

of the excess emission. We have identified 13 new excess emission stars above a $2 \sigma$ threshold. We are continuing IRSF observations, for the rest of our 734 samples.

\section{SUMMARY}

Using the AKARI/MIR All-Sky PSC, we are searching for new debris disks. Instead of using 2MASS fluxes, which have large errors, we performed follow-up observations with IRSF to obtain accurate fluxes in the J-, $\mathrm{H}-$, and Ks-bands. As a result, we have succeeded in improving the SEDs of the central stars. This improvement allows us to make more reliable selection of the debris disks candidates.

\section{ACKNOWLEDGEMENTS}

This work was supported by Grants-in-Aid for Scientific Research (24740122) from the Japan Society for the Promotion of Science (JSPS), and the Nagoya University Global Center of Excellence Program, "Quest for Fundamental Principles in the Universe" (QFPU) from JSPS and the Ministry of Education, Culture, Sports, Science and Technology of Japan.

\section{REFERENCES}

Fujiwara, H., et al., 2010, Silica-rich Bright Debris Disk around HD 15407A, ApJ, 749, L29

Ishihara, D., et al., 2010, The AKARI/IRC MidInfrared All-Sky Survey, A\&A, 514, 1

Kurucz, R. L., 2013, Model Atmospheres for Population Synthesis, IAUS, 149, 225

Wright, C. O., et al., 2003, The Tycho-2 Spectral Type Catalog, AJ, 125, 359

Wyatt, M. C., 2005, Dust in Resonant Extrasolar Kuiper Belts: Grain Size and Wavelength Dependence of Disk Structure, ApJ, 639, 1153 